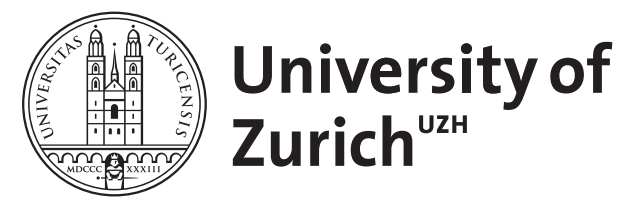

\title{
Nonconvex regularization in remote sensing
}

Tuia, Devis ; Flamary, Rémi ; Barlaud, Michel

\begin{abstract}
In this paper, we study the effect of different regularizers and their implications in highdimensional image classification and sparse linear unmixing. Although kernelization or sparse methods are globally accepted solutions for processing data in high dimensions, we present here a study on the impact of the form of regularization used and its parameterization. We consider regularization via traditional squared (!2) and sparsity-promoting (!1) norms, as well as more unconventional non convex regularizers (!p and log sum penalty). We compare their properties and advantages on several classification and linear unmoving tasks and provide advices on the choice of the best regularizer for the problemat hand. Finally, we also provide a fully functional toolbox for the community.
\end{abstract}

DOI: https://doi.org/10.1109/TGRS.2016.2585201

Posted at the Zurich Open Repository and Archive, University of Zurich ZORA URL: https://doi.org/10.5167/uzh-126406

Journal Article

Accepted Version

Originally published at:

Tuia, Devis; Flamary, Rémi; Barlaud, Michel (2016). Nonconvex regularization in remote sensing. IEEE Transactions on Geoscience and Remote Sensing, 54(11):6470-6480.

DOI: https://doi.org/10.1109/TGRS.2016.2585201 


\title{
Nonconvex Regularization in Remote Sensing
}

\author{
Devis Tuia, Senior Member, IEEE, Rémi Flamary, and Michel Barlaud, Senior Member, IEEE
}

\begin{abstract}
In this paper, we study the effect of different regularizers and their implications in high-dimensional image classification and sparse linear unmixing. Although kernelization or sparse methods are globally accepted solutions for processing data in high dimensions, we present here a study on the impact of the form of regularization used and its parameterization. We consider regularization via traditional squared $\left(\ell_{2}\right)$ and sparsity-promoting $\left(\ell_{1}\right)$ norms, as well as more unconventional nonconvex regularizers $\left(\ell_{p}\right.$ and $\log$ sum penalty). We compare their properties and advantages on several classification and linear unmixing tasks and provide advices on the choice of the best regularizer for the problem at hand. Finally, we also provide a fully functional toolbox for the community.
\end{abstract}

Index Terms-Classification, hyperspectral, nonconvex, regularization, remote sensing, sparsity, unmixing.

\section{INTRODUCTION}

$\mathbf{R}$ EMOTE sensing image processing [1] is a fast-moving area of science. Data acquired from satellite or airborne sensors and converted into useful information (land cover maps, target maps, mineral compositions, and biophysical parameters) have nowadays entered many applicative fields: efficient and effective methods for such conversion are therefore needed. This is particularly true for data sources such as hyperspectral and very high resolution images, whose data volume is big and structure is complex: for this reason, many traditional methods perform poorly when confronted to this type of data. The problem is even more exacerbated when dealing with multisource and multimodal data, representing different views of the land being studied (different frequencies, different seasons, angles, etc.). This created the need for more advanced techniques, often based on statistical learning [2].

Among such methodologies, regularized methods are certainly the most successful. Using a regularizer imposes some constraints on the class of functions to be preferred during the optimization of the model and can thus be beneficial if we know what these properties are. More often, regularizers are used to favor simpler functions over very complex ones in order to avoid overfitting of the training data: in classification,

Manuscript received April 11, 2016; revised June 2, 2016; accepted June 22, 2016. This work was supported in part by the Swiss National Science Foundation under Grant PP00P2-150593. D. Tuia and R. Flamary contributed equally to this paper.

D. Tuia is with the University of Zurich, 8006 Zurich, Switzerland (e-mail: devis.tuia@geo.uzh.ch).

R. Flamary is with Université Côte d'Azur, OCA, CNRS, Lagrange, 06000 Nice, France (e-mail: remi.flamary@unice.fr).

M. Barlaud is with Université Côte d'Azur, CNRS, I3S, 06000 Nice, France. Color versions of one or more of the figures in this paper are available online at http://ieeexplore.ieee.org.

Digital Object Identifier 10.1109/TGRS.2016.2585201 the support vector machine uses this form of regularization [3], [4], while in regression, examples can be found in kernel ridge regression or Gaussian processes [5].

However, smoothness-promoting regularizers are not the only ones that can be used: depending on the properties one wants to promote, other choices are becoming more and more popular. A first success story is the use of Laplacian regularization [6]: by enforcing smoothness in the local structure of the data, one can promote the fact that points that are similar in the input space must have a similar decision function (Laplacian SVM [7], [8] and dictionary-based methods [9], [10]) or be projected close after a feature extraction step (Laplacian eigenmaps [11] and manifold alignment [12]). Another popular property to be enforced, on which we will focus the rest of this paper, is sparsity [13]. Sparse models have only a part of the initial coefficients which is active (i.e., nonzero) and are thus compact. This is desirable in classification when the dimensionality of the data is very high (e.g., when adding many spatial filters [14], [15] or using convolutional neural networks [16], [17]) or in sparse coding when we need to find a relevant dictionary to express the data [18]. Even though nonsparse models can work well in terms of overall accuracy, they still store information about the training samples to be used at test time: if such information is very high dimensional and the number of training samples is important, the memory requirements, the model complexity, and - as a consequence - the execution time are strongly affected. Therefore, when processing next-generation large data using models generating millions of features [19], [20], sparsity is very much needed to make models portable while remaining accurate. For this reason, sparsity has been extensively used in the following: 1) spectral unmixing [21], where a large variety of algorithms is deployed to select endmembers as a small fraction of the existing data [18], [22], [23]; 2) image classification, where sparsity is promoted to have portable models either at the level of the samples used in reconstruction-based methods [24], [25] or in feature selection schemes [15], [26], [27]; and 3) and more focused applications such as 3-D reconstruction from SAR [28], phase estimation [29], or pansharpening [30].

A popular approach to recover sparse features is to solve a convex optimization problem involving the $\ell_{1}$ norm (or Lasso) regularization [31] $-[33]$. Proximal splitting methods have been shown to be highly effective in solving sparsity-constrained problems [34]-[36]. The Lasso formulation based on the penalty on the $\ell_{1}$ norm of the model has been shown to be an efficient shrinkage and sparse model selection method in regression [37]-[39]. However, the Lasso regularizer is known to promote biased estimators, leading to suboptimal classification performances when strong sparsity is promoted [40], [41]. A way out of this dilemma between sparsity and performance is to retrain a classifier, this time nonsparse, after the feature 
selection has been performed with Lasso [15]. Such scheme works but at the price of training a second model, thus leading to extra computational effort and to the risk of suboptimal solutions, since we are training a model with the features that were considered optimal by another. In unmixing, synthetic examples also show that the $\ell_{1}$ regularization is not the one leading to the best abundance estimation [42].

In recent years, there has been a trend in the study of unbiased sparse regularizers. These regularizers, typically the $\ell_{0}, \ell_{q}$, and log sum penalty (LSP) [40], can solve the dilemma between sparsity and performance but are nonconvex and therefore cannot be solved by known off-the-shelf convex optimization tools. Therefore, such regularizers have until now received little attention in the remote sensing community. A handful of papers using $\ell_{q}$ norm is found in the field of spectral unmixing [42]-[44], where authors consider nonnegative matrix factorization solutions; in the modeling of electromagnetic induction responses, where the model parameters were estimated by regularized least squares estimation [45]; in feature extraction using deconvolutional networks [46]; and in structured prediction, where authors use a nonconvex sparse classifier to provide posterior probabilities to be used in a graph cut model [47]. In all of these studies, the nonconvex regularizer outperformed the $\ell_{1}$ while still providing sparse solutions.

In this paper, we give a critical explanation and theoretical motivations for the success of regularized classification, with a focus on nonconvex methods. By comparing it with other traditional regularizers (ridge $\ell_{2}$ and Lasso $\ell_{1}$ ), we advocate the use of nonconvex regularization in remote sensing image processing tasks: nonconvex optimization marries the advantages of accuracy and sparsity in a single model, without the need of unbiasing in two steps or reducing the level of sparsity to increase performance. We also provide a freely available toolbox for the interested readers that would like to enter this growing field of investigation.

The remainder of this paper is organized as follows. In Section II, we present a general framework for regularized remote sensing image processing and discuss different forms of convex and nonconvex regularization. We will also present the optimization algorithm proposed. Then, in Section III, we apply the proposed nonconvex regularizers to the problem of multiand hyperspectral image classification and therefore present the specific data term for classification and study it in synthetic and real examples. In Section IV, we apply our proposed framework to the problem of linear unmixing, present the specific data term for unmixing, and study the behavior of the different regularizers in simulated examples involving true spectra from the USGS library. Section V concludes this paper.

\section{Optimization AND NONCONVEX REgulaRizATION}

In this section, we give an intuitive explanation of regularized models. We first introduce the general problem of regularization and then explore convex and nonconvex regularization schemes, with a focus on sparsity-inducing regularizers. Finally, we present the optimization algorithms to solve nonconvex regularization, with accent put on proximal splitting methods such as general iterative shrinkage and thresholding (GIST) [48].
TABLE I

DEFINITION OF THE REgULARIZATION TERMS CONSIDERED

\begin{tabular}{|l|c|}
\hline Regularization term & $g\left(\left|w_{k}\right|\right)$ \\
\hline Ridge, $\ell_{2}$ norm & $\left|w_{k}\right|^{2}$ \\
Lasso, $\ell_{1}$ norm & $\left|w_{k}\right|$ \\
Log sum penalty (LSP) & $\log \left(\left|w_{k}\right| / \theta+1\right)$ \\
$\ell_{p}$ with $0<p<1$ & $\left|w_{k}\right|^{p}$ \\
\hline
\end{tabular}

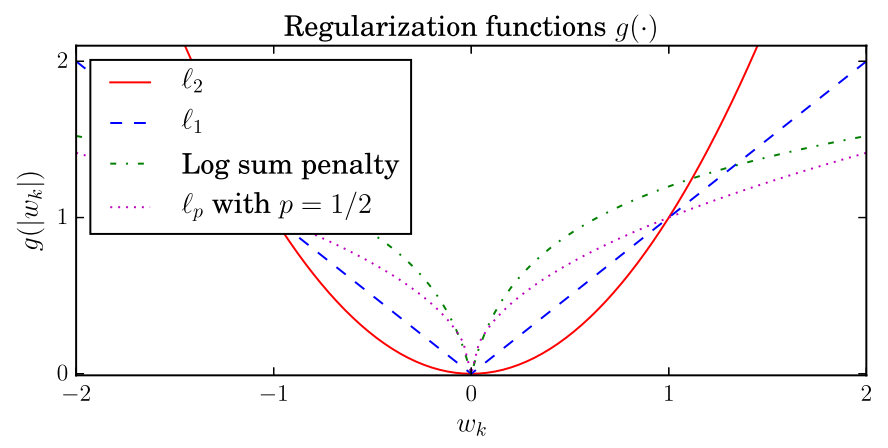

Fig. 1. Illustration of the regularization terms $g(\cdot)$. Note that both $\ell_{2}$ and $\ell_{1}$ regularizations are convex and that LSP and $\ell_{p}$ with $p=1 / 2$ are concave on their positive orthant.

\section{A. Optimization Problem}

Regularized models address the following problem:

$$
\min _{\mathbf{w} \in \mathbb{R}^{d}} L(\mathbf{w})+\lambda R(\mathbf{w})
$$

where $L(\cdot)$ is a smooth function (Lipschitz gradient), $\lambda>0$ is a regularization parameter, and $R(\cdot)$ is a regularization function. This kind of problem is extremely common in data mining, denoising, and parameter estimation.

$L(\cdot)$ is often an empirical loss that measures the discrepancy between a model $\mathbf{w}$ and a data set containing real-life observations.

The regularization term $R(\cdot)$ is added to the optimization problem in order to promote a simple model, which has been shown to lead to a better estimation [49]. All of the regularization terms discussed in this paper are of the form

$$
R(\mathbf{w})=\sum_{k} g\left(\left|w_{k}\right|\right)
$$

where $g$ is a monotonically increasing function. This means that the complexity of the model $\mathbf{w}$ can be expressed as a sum of the complexity of each feature $k$ in the model.

The specific form of the regularizer will change the assumptions made on the model. In the following, we discuss several classes of regularizers of increasing complexity: differentiable, nondifferentiable (i.e., sparsity inducing), and, finally, both nondifferentiable and nonconvex. A summary of all of the regularization terms investigated in this paper is given in Table I, along with an illustration of the regularization as a function of the value of the coefficient $w_{k}$ (Fig. 1).

\section{B. Nonsparse Regularization}

One of the most common regularizers is the square $\ell_{2}$ norm of model $\mathbf{w}$, i.e., $R(\mathbf{w})=\|\mathbf{w}\|^{2}\left(g(\cdot)=(\cdot)^{2}\right)$. This regularization will penalize large values in the vector $\mathbf{w}$ but is isotropic, i.e., it will not promote a given direction for the vector $\mathbf{w}$. This regularization term is also known as $\ell_{2}$, quadratic, or ridge 
regularization and is commonly used in linear regression and classification. For instance, logistic regression is often regularized with a quadratic term. Also, note that the support vector machine is regularized using the $\ell_{2}$ norm in the reproducing kernel Hilbert space of the form $R(\mathbf{w})=\mathbf{w}^{\top} \mathbf{K} \mathbf{w}$ [50].

\section{Sparsity-Promoting Regularization}

In some cases, not all of the features or observations are of interest for the model. In order to get a better estimation, one wants the vector $\mathbf{w}$ to be sparse, i.e., to have several components exactly 0 . For linear prediction, sparsity in the model $\mathbf{w}$ implies that not all features are used for the prediction. ${ }^{1}$ This means that the features showing a nonzero value in $w_{k}$ are then "selected." Similarly, when estimating a mixture, one can suppose that only few materials are present, which again implies sparsity of the abundance coefficients.

In order to promote sparsity in $\mathbf{w}$, one needs to use a regularization term that increases when the number of active components grows. The obvious choice is to use the $\ell_{0}$ pseudonorm that returns directly the number of nonzero coefficients in $\mathbf{w}$. Nevertheless, the $\ell_{0}$ term is nonconvex and nondifferentiable and cannot be optimized exactly unless all of the possible subsets are tested. Despite recent works aiming at solving directly this problem via discrete optimization [51], this approach is still computationally impossible even for medium-sized problems. Greedy optimization methods have been proposed to solve this kind of optimization problem and have led to efficient algorithms such as orthogonal matching pursuit (OMP) [52] or orthogonal least square (OLS) [53]. However, one of the most common approaches to promote sparsity without recurring to the $\ell_{0}$ regularizer is to use the $\ell_{1}$ norm instead. This approach, also known as the Lasso in linear regression, has been widely used in compressive sensing in order to estimate with precision a few components in a large sparse vector.

Now, we discuss the intuition why using a regularization term such as $\ell_{1}$ promotes sparsity. The reason behind the sparsity of the $\ell_{1}$ norm lies in the nondifferentiability at 0 shown in Fig. 1 (dashed blue line). For the sake of readability, we will suppose here that $R(\cdot)$ is convex, but the intuition is the same, and the results can be generalized to the nonconvex functions presented in the next section. For a more illustrative example, we use a 1-D comparison between the $\ell_{2}$ and $\ell_{1}$ regularizers (Fig. 2).

- When both the data and regularization term are differentiable, a stationary point $\mathbf{w}^{\star}$ has the following property:

$$
\nabla L\left(\mathbf{w}^{\star}\right)+\lambda \nabla R\left(\mathbf{w}^{\star}\right)=\mathbf{0} .
$$

In other words, the gradients of both functions have to cancel themselves exactly. This is true for the $\ell_{2}$ regularizer everywhere, but also for the $\ell_{1}$, with the exception of $w_{k}=0$. If we consider the $\ell_{2}$ regularizer as an example (left plot in Fig. 2), we see that each point has a specific gradient, corresponding to the tangent to each point (e.g., the red dashed line). The stationary point is reached in this case for $w_{k}=0$, as given by the black line at the left plot of Fig. 2.

\footnotetext{
${ }^{1}$ Note that zero coefficients might happen also in the $\ell_{2}$ solution, but the regularizer itself does not promote their appearance.
}

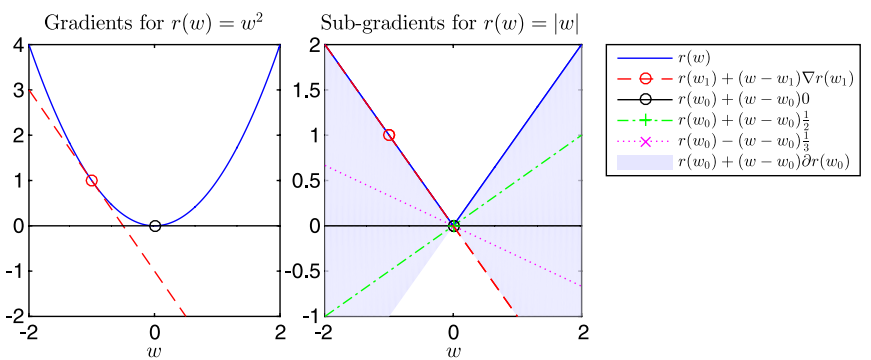

Fig. 2. Illustration of gradients and subgradients on differentiable $\ell_{2}$ (left) and nondifferentiable $\ell_{1}$ (right) functions.

- When the second term in (3) is not differentiable (as in the $\ell_{1}$ case at 0 presented at the right plot of Fig. 2), the gradient is not unique anymore, and one has to use the subgradients and subdifferentials. For a convex function $R(\cdot)$, a subgradient at $\mathbf{w}^{t}$ is a vector $\mathbf{x}$ such that $R(\mathbf{w}) \geq$ $\mathbf{x}^{\top}\left(\mathbf{w}-\mathbf{w}^{t}\right)+R\left(\mathbf{w}^{t}\right)$, i.e., it is the slope of a linear function that remains below the function. In 1-D, a subgradient defines a line touching the function at the nondifferentiable point (in the case of Fig. 2, at 0) but stays below the function everywhere else, e.g., the black and green dotted-dash lines in Fig. 2 (right). The subdifferential $\partial R\left(\mathbf{w}^{t}\right)$ is the set of all of the subgradients that respect the aforementioned minoration relation. The subdifferential is illustrated in Fig. 2 by the area in light blue, which contains all possible solutions.

Now the optimality constraints cannot rely on equality since the subgradient is not unique, which leads to the following optimality condition:

$$
\mathbf{0} \in \nabla L\left(\mathbf{w}^{\star}\right)+\lambda \partial R\left(\mathbf{w}^{\star}\right) .
$$

This is very interesting in our case because this condition is much easier to satisfy than (3). Indeed, we just need to have a single subgradient in the whole subdifferential $\partial R(\cdot)$ that can cancel the gradient $\nabla L(\cdot)$. In other words, only one of the possible straight lines in the blue area is needed to cancel the gradient, thus making the chances for a null coefficient much higher. For instance, when using the $\ell_{1}$ regularization, the subdifferential of variable $w_{k}$ in 0 is the set $[-\lambda, \lambda]$. When $\lambda$ becomes large enough, it is larger than all of the components of the gradient $\nabla L(\cdot)$, and the only solution verifying the conditions is the null vector $\mathbf{0 .}$

The $\ell_{1}$ regularization has been largely studied. Because it is convex, it means that it avoids the problem of local minima, and many efficient optimization procedures exist to solve it (e.g., LARS [54] and forward backward splitting [55]). However, the sparsity of the solution using $\ell_{1}$ regularization often comes with a cost in terms of generalization. While theoretical studies show that, under some constraint, the $\ell_{1}$ can recover the true relevant variables and their sign, the solution obtained will be biased toward 0 [56]. Fig. 3 illustrates the bias in a two-class toy data set: the $\ell_{1}$ decision function (red line) is biased with respect to the Bayes decision function (blue line). In this case, the bias corresponds to a rotation of the separating hyperplane. In practice, one can deal with this bias by estimating again the 


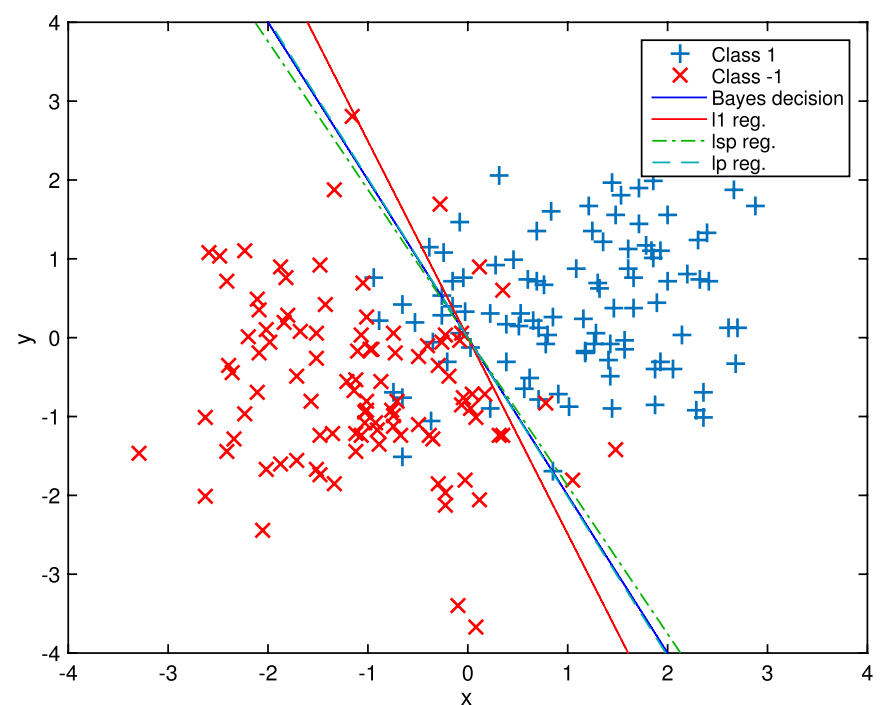

Fig. 3. Example of a two-class toy example with 2 discriminant features and 18 noisy features. The regularization parameter of each method has been chosen as the minimal value that leads to the correct sparsity with only two features selected.

model on the selected subset of variables using an isotropic norm (e.g., $\ell_{2}$ ) [15], but this requires us to solve again an optimization problem. The approach that we propose in this paper is to use a nonconvex regularization term that will still promote sparsity while minimizing the aforementioned bias. To this end, we present nonconvex regularization in the next section.

\section{Nonconvex Regularization}

In order to promote more sparsity while reducing the bias, several works have looked at nonconvex yet continuous regularization. Such regularizers have been proposed, for instance, in statistical estimation [57], compressed sensing [40], or machine learning [41]. Popular examples are the smoothly clipped absolute deviation [57], the minimax concave penalty [58], and the LSP [40] considered in the following (see [48] for more examples). In the following, we will investigate two of them in more detail: $\ell_{p}$ pseudonorm with $p=1 / 2$ and LSP, both also displayed in Fig. 1.

All of the aforementioned nonconvex regularizations share some particular characteristics that make them of interest in our case. First (and as the $\ell_{0}$ pseudonorm and $\ell_{1}$ norm), they all have a nondifferentiability in $\mathbf{0}$, which - as we have seen in the previous section-promotes sparsity. Second, they are all concave in their positive orthant, which limits the bias because their gradient will decrease for large values of $w_{k}$ limiting the shrinkage (as compared to the $\ell_{1}$ norm, whose gradient for $w_{k} \neq 0$ is constant). Intuitively, this means that, with a nonconvex regularization, it will become more difficult for large coefficients to be shrunk toward 0 because their gradient is small. On the contrary, the $\ell_{1}$ norm will treat all coefficients equally and apply the same attraction to the stationary point to all of them. The decision functions for the LSP and $\ell_{p}$ norms are shown in Fig. 3 and are much closer to the actual (true) Bayes decision function.

\section{E. Optimization Algorithms}

Owing to the differentiability of the $L(\cdot)$ term, the optimization problem can be solved using proximal splitting methods [55]. The convergence of those algorithms to a global minimum is well studied in the convex case. For nonconvex regularization, recent works have proved that proximal methods can be used with nonconvex regularizers when a simple closed-form solution of the proximity operator for the regularization can be computed [48]. Authors in [59] have studied the convergence to a local stationary point of proximal methods with nonconvex regularization for several loss functions.

In this paper, we used the GIST algorithm proposed in [48]. This approach is a first-order method that consists in iteratively linearizing $L(\cdot)$ in order to solve very simple proximal operators at each iteration. At each iteration $t+1$, one computes the model update $w^{t+1}$ by solving

$$
\min _{\mathbf{w}} \nabla L\left(\mathbf{w}^{t}\right)^{\top}\left(\mathbf{w}-\mathbf{w}^{t}\right)+\lambda R(\mathbf{w})+\frac{\mu}{2}\left\|\mathbf{w}-\mathbf{w}^{t}\right\|_{2}^{2} .
$$

When $\mu$ is a Lipschitz constant of $L(\cdot)$, the aforementioned cost function is a majorization of $L(\cdot)+\lambda R(\cdot)$ which ensures a decrease of the objective function at each iteration. Problem (5) can be reformulated as a proximity operator

$$
\operatorname{prox}_{\lambda R}(\mathbf{v})=\arg \min _{\mathbf{w}} \quad \lambda R(\mathbf{w})+\frac{\mu}{2}\left\|\mathbf{w}-\mathbf{v}^{t}\right\|_{2}^{2}
$$

where $\mathbf{v}^{t}=\mathbf{w}^{t}-(1 / \mu) \nabla L\left(\mathbf{w}^{t}\right)$ can be seen as a gradient step w.r.t. $L(\cdot)$ followed by a proximal operator at each iteration. Note that the efficiency of a proximal algorithm depends on the existence of a simple closed-form solution for solving the proximity operator in (6). Luckily, numerous operators exist in the convex case (detailed list in [55]), and some nonconvex proximal operators can be computed on the regularization used in our work (see [48, Appendix 1] for LSP and [60, eq. 11] for $\ell_{p}$ with $p=1 / 2$ ). Note that efficient methods which estimate the Hessian matrix [61], [62] exist, as well as a wide range of methods based on DC programming, which have shown to work very well in practice [62], [63] and can handle the general case $p \in(0,1]$ for the $\ell_{p}$ pseudonorm (see [64] for an implementation).

Finally, when one wants to perform variable selection using the $\ell_{0}$ pseudonorm as regularization, the exact solution of the combinatorial problem is not always necessary. As mentioned previously, greedy optimization methods have been proposed to solve these optimization problems and have led to efficient algorithms as OMP [52] or OLS [53]. In this paper, we will not consider these methods in detail, but they have been shown to perform well on least square minimization problems.

\section{Classification With Feature Selection}

In this section, we tackle the problem of sparse classification. Through a toy example and a series of real-data experiments, we will study the interest of nonconvex regularization.

\section{A. Model}

The model that we will consider in the experiments is a simple linear classifier of the form $f(\mathbf{x})=\mathbf{w}^{\top} \mathbf{x}+b$, where $\mathbf{w} \in \mathbb{R}^{d}$ is the normal vector to the separating hyperplane and $b$ 
is a bias term. In the binary case $\left(y_{i} \in[-1 ; 1]\right)$, the estimation is performed by solving the regularized optimization problem

$$
\min _{\mathbf{w}, b} \frac{1}{n} \sum_{i=1}^{n} \mathcal{L}\left(y_{i}, f\left(\mathbf{x}_{i}\right)\right)+R(\mathbf{w})
$$

where $R(\mathbf{w})$ is one of the regularizers in Table I and $\mathcal{L}\left(y_{i}, f\left(\mathbf{x}_{i}\right)\right)$ is a classification loss that measures the discrepancy between the prediction $f\left(\mathbf{x}_{i}\right)$ and the true label $y_{i}$. Hereafter, we will use the squared hinge loss

$$
\mathcal{L}\left(y_{i}, f\left(\mathbf{x}_{i}\right)\right)=\max \left(0,1-y_{i} f\left(\mathbf{x}_{i}\right)\right)^{2} .
$$

When dealing with multiclass problems, we use a oneagainst-all procedure, i.e., we learn one linear function $f_{j}(\cdot)$ per class $j$ and then predict the final class for a given observed pixel $\mathbf{x}$ as the solution of $\arg \min _{j} f_{j}(\mathbf{x})$. In practice, this leads to an optimization problem similar to (7), where we need to estimate a matrix $\mathbf{W}$, containing the coefficients per each class. The number of coefficients to be estimated is therefore the size $d$ of the input space multiplied by the number of classes $C$ plus one bias coefficient per class.

\section{B. Toy Example}

First, we consider in detail the toy example in Fig. 3: the data considered are 20-dimensional, where the first two dimensions are discriminative (they correspond to those plotted in Fig. 3), while the others are not (they are generated as Gaussian noise). The correct solution is therefore to assign nonzero coefficients to the two discriminative features and $w_{k}=0$ for all of the others.

Fig. 3 shows the classifiers estimated for the smallest value of the regularization term $\lambda$, which leads to the correct sparsity level (two features selected). This ensures that we have selected the proper components while minimizing the bias for all methods. This also illustrates that the $\ell_{1}$ classifier has a stronger bias (i.e., provides a decision function further away from the optimal Bayes classifier) than the classifiers regularized by nonconvex functions.

Let us now focus on the effect of the regularization term and of its strength, defined by the regularization parameter $\lambda$ in (7). Fig. 4 illustrates a regularization path, i.e., all of the solutions obtained by increasing the regularization parameter $\lambda .^{2}$ Each line corresponds to one input variable, and those with the largest coefficients (and in color) are the discriminative ones. Considering the $\ell_{2}$ regularization (top left panel in Fig. 4), no sparsity is achieved, and even if the two correct features have the largest coefficients, the solution is not compact. The $\ell_{1}$ solution (top right panel) shows a correct sparse solution for $\lambda=10^{-1}$ (vertical black line, where all of the coefficients but two are 0), but the smallest coefficient is biased (it is smaller than expected by the Bayes classifier, represented by the horizontal dashed lines). The two nonconvex regularizers (bottom line of Fig. 4) show the correct features selected but

\footnotetext{
${ }^{2}$ A "regularization path" for the $\ell_{1}$ is generally computed using homotopy algorithms [65]. However, experiments show that the computational complexity of the complete $\ell_{1}$ path remains high for high-dimensional data. Therefore, in our experiments, we used an approximate path (i.e., a discrete sampling of $\lambda$ values along the path).
}
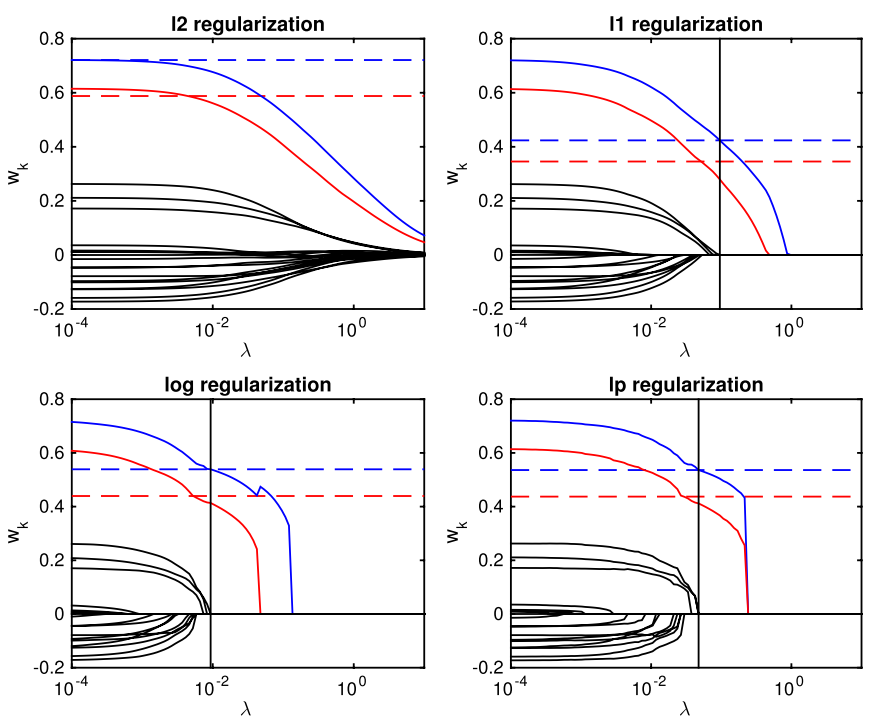

Fig. 4. Regularization paths for the toy example in Fig. 3. Each line corresponds to the coefficients $w_{k}$ attributed to each feature along the different values of $\lambda$. The best fit is met for each regularizer at the black vertical line, where all coefficients but two are 0 . The unbiased Bayes classifier coefficients (the correct coefficients) are represented by the horizontal dashed lines.

a smaller bias: the coefficient retrieved is closer to the optimal ones of the Bayes classifier. Moreover, the nonzero coefficients stay close to the correct values for a wider set of regularization parameters and then drop directly to zero: this means that the nonconvex model either does not have enough features to train or has little features with the right coefficients, contrary to the $\ell_{1}$ that can retrieve a sparse solution with wrong coefficients, as seen in the part to the right of the vertical black line of the $\ell_{1}$ regularization path.

\section{Remote Sensing Images}

Data: The real data sets considered are three very high resolution remote sensing images.

1) Thetford mines. The first data set is acquired over the Thetford mines site in Québec, Canada, and contains two data sources: a VHR color image (three channels, red-green-blue) at $20-\mathrm{cm}$ resolution and a long wave infrared (LWIR, 84 channels) hyperspectral image at approximately $1-\mathrm{m}$ resolution. ${ }^{3}$ The LWIR images are downsampled by a factor 5 to match the resolution of the RGB data, leading to a $(4386 \times 3769 \times 87)$ datacube. A 7 -classes ground truth is available. The RGB composite, band 1 of te LWIR data and train/test ground truths are provided in Fig. 5.

2) Houston. The second image is a CASI image acquired over Houston with 144 spectral bands at $2.5-\mathrm{m}$ resolution. A field survey is also available (14703 labeled pixels, divided in 14 land use classes). A LiDAR DSM was also available and was used as an additional feature. ${ }^{4}$ The CASI

\footnotetext{
${ }^{3}$ The data were proposed as the Data Fusion Contest 2014 [66] and are available on the IADF TC website for download http://www.grss-ieee.org/ community/technical-committees/data-fusion/.

${ }^{4}$ The data were proposed as the Data Fusion Contest 2013 [67] and are available on the IADF TC website for download http://www.grss-ieee.org/ community/technical-committees/data-fusion/.
} 
(a)
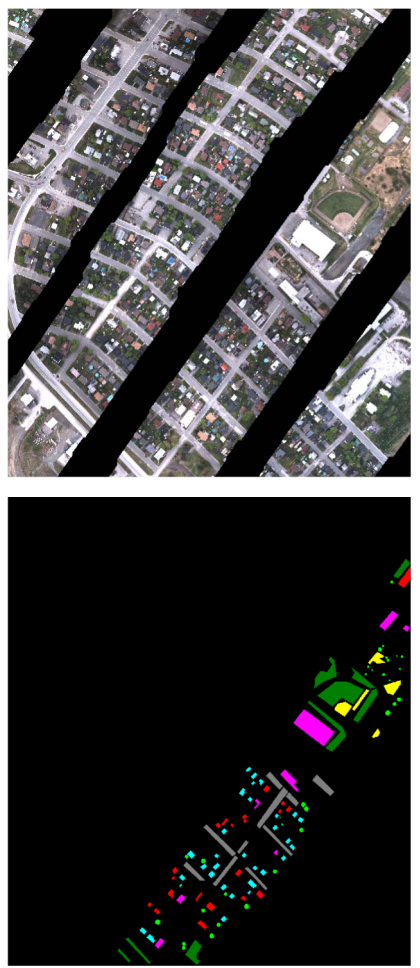

(c) (b)
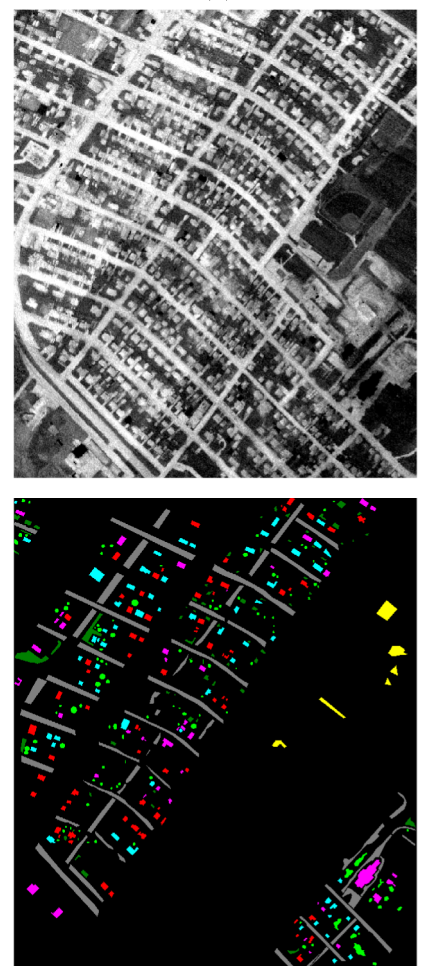

(d)
Fig. 5. Thetford mines 2014 data set used in the classification experiments, along with its labels. (a) RGB. (b) LWIR band 1. (c) Ground truth training. (d) Ground truth test.
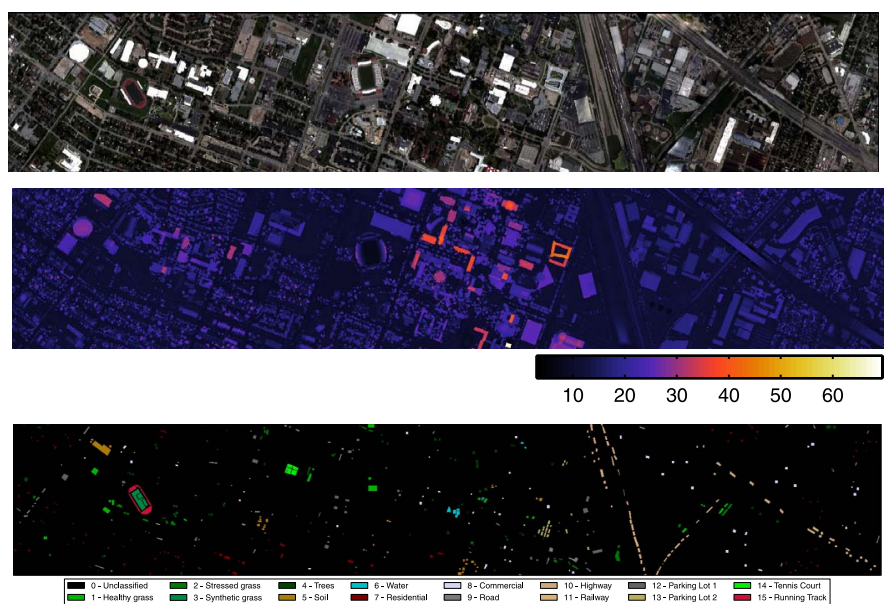

Fig. 6. Houston data set used in the classification experiments. (Top) True color representation of the hyperspectral image (144 bands). (Middle) Detrended LiDAR DSM. (Bottom) Labeled samples (all of the available ones, in 15 classes).

image was corrected with histogram matching for a large shadowed part at the right side (as in [27]), and the DSM was detrended by a 3-m trend at the left-right direction. Image, DSM, and ground truth are illustrated in Fig. 6.

3) Zurich summer. The third data set is a series of 20 QuickBird tiles issued from a single image acquired over the city of Zurich, Switzerland, in August 2002..$^{5}$ The data have been pansharpened at $0.6-\mathrm{m}$ spatial resolution, and

${ }^{5}$ The data set is freely available at https://sites.google.com/site/ michelevolpiresearch/data/zurich-dataset/.
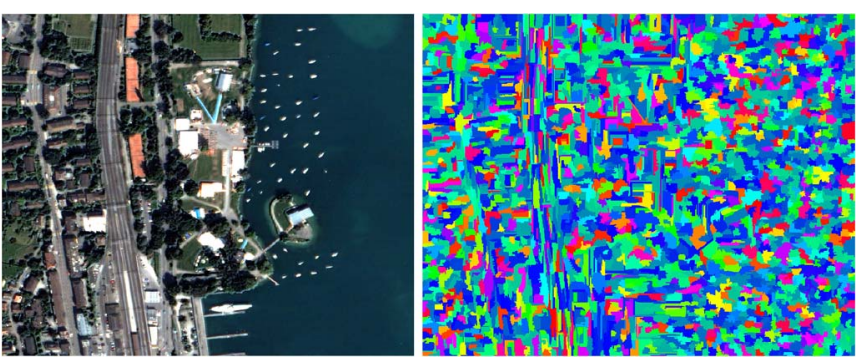

Fig. 7. Example on tile \#3 of the superpixels extracted by the Felzenszwalb algorithm [69].

a dense ground truth is provided for each image. Eight classes are depicted: buildings, roads, railway, water, swimming pools, trees, meadows, and bare soil. More information on the data can be found in [68]. To reduce computational complexity, we extracted a set of superpixels using the Felzenszwalb algorithm [69], which reduced the number of samples from $\sim 10^{6}$ pixels per image to a few thousands. An example of the superpixels extracted on image tile \#3 is given in Fig. 7.

Setup: For all data sets, contextual features were added to the spectral bands in order to improve the geometric quality of classification [14]: morphological and texture filters were added, following the list in [15]. Each image was processed to extract the most effective filters for its processing.

- For the Thetford mines data set, the filters were extracted from the RGB image and from a normalized ratio between the red band and the average of the LWIR bands (following the strategy of the winners of the 2014 Data Fusion Contest [66]), which approaches a vegetation index. Given the extremely high resolution of the data set, the filters were computed with the size range $\{7, \ldots 23\}$, leading to 100 spatial features.

- For the Houston case, the filters were calculated on the three first principal component projections extracted from the hyperspectral image and on the DSM. Given the smaller resolution of this data set, the convolution sizes of the local filters are in the range $\{3, \ldots, 15\}$ pixels. This leads to 240 spatial features.

- For the Zurich summer data set, spatial filters were computed directly on the four spectral bands, plus the NDVI and the NDWI indices. Then, average, minimum, maximum, and standard deviation values per superpixel were extracted as feature values. Since the spatial resolution is comparable to the one of the Houston data sets, the same sizes of convolution filters are used, leading to a total of 360 spatial features.

The joint spatial-spectral input space is obtained by stacking the original images to the spatial filters above. It is therefore of dimension 188 in the Thetford mines data, 384 in the Houston data, and 366 in the Zurich summer case.

Regarding the classifier, we considered the linear classifier of (7) with a squared hinge loss.

- In the Thetford mines case, we use 5000 labeled pixels per class. Given the spatial resolution of the image and 

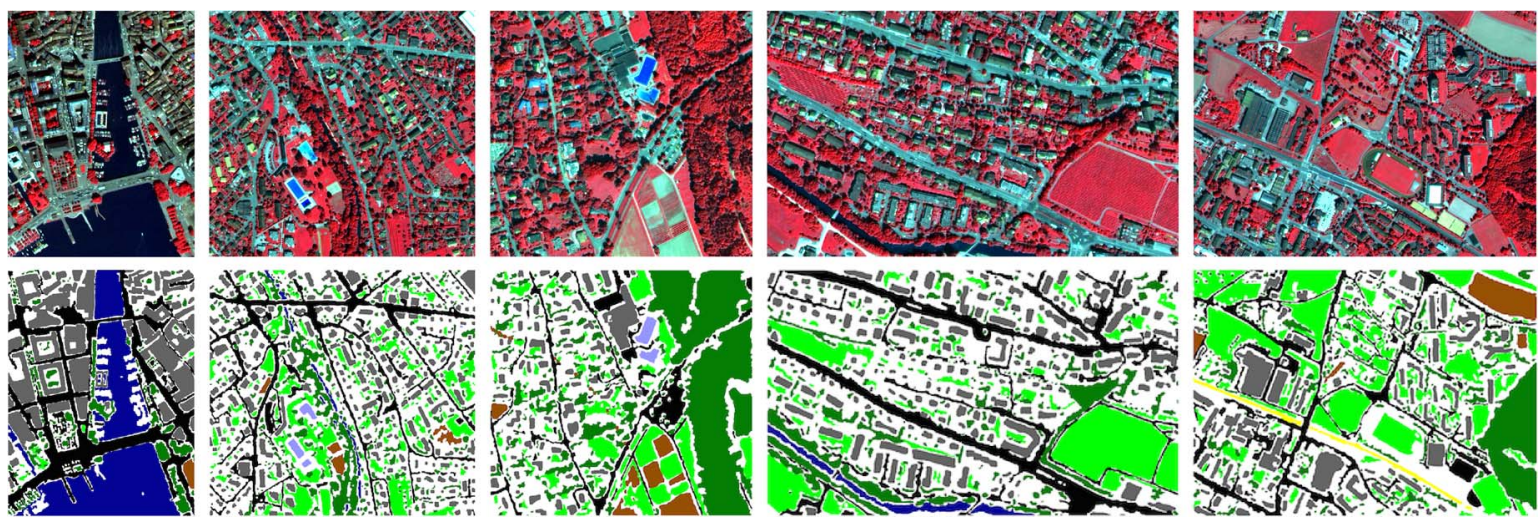

Fig. 8. Five test images of the Zurich summer data set (from left to right, tiles \#16 to \#20), along with their ground truth.
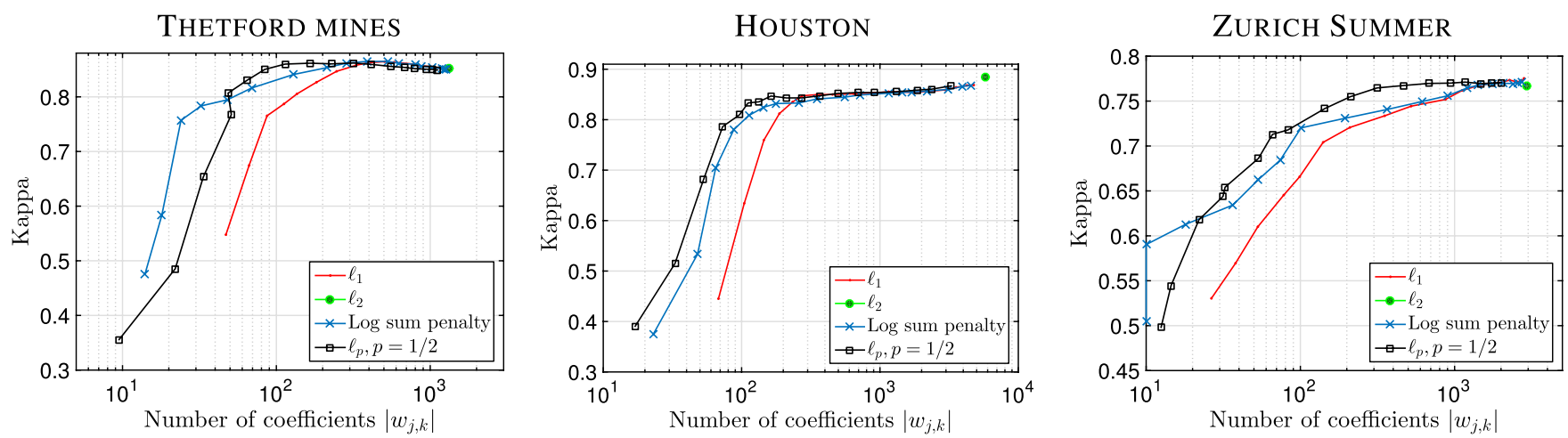

Fig. 9. Performance (kappa) versus compactness (number of coefficients $w_{j, k}>0$ ) for the different regularizers in the Thetford mines, Houston, and Zurich summer data sets.

the 568242 labeled points available in the training ground truth, this only represents approximately $5 \%$ of the labeled pixels in the training image. For the test, we use the entire test ground truth, which is spatially disconnected to the training one (except for the class "soil"; see Fig. 5) and carries 1.5 million labeled pixels.

- In the Houston case, we also proceed with pixel classification. All of the models are trained with 60 labeled pixels per class, randomly selected, from the available training data. We report performances on the entire test set provided in the Data Fusion Contest 2013, which is spatially disconnected from the training set (Fig. 6).

- For the Zurich summer data, we deal with superpixels and 20 separate images. We used images \#1-15 to train the classifier and then tested on the five remaining images (Fig. 8). Given the complexity of the task (not all of the images have all of the classes and the urban fabrics depicted vary from scene to scene), we used $90 \%$ of the available superpixels in the 15 training images, which resulted in 30649 superpixels. All of the labeled superpixels in the test images (8960 superpixels) are used as test set.

Regarding the regularizers, we compare the four regularizers of Table I $\left(\ell_{1}, \ell_{2}, \mathrm{LSP}\right.$, and $\ell_{p}$ with $\left.p=1 / 2\right)$ and study the joint behavior of accuracy and sparsity along a regularization path, i.e., for different values of $\lambda=\left\{1 e^{-5}, \ldots, 1 e^{-1}\right\}$, with 18 steps. For each step, the experiment was repeated ten times with different train/test sets (each run with the same training samples for all regularizers), and the average kappa and number of active coefficients are reported in Fig. 9. Also, note that we report the total number of coefficients in the multiclass case $\left|w_{j, k}\right|$, which is equal to the number of features multiplied by the number of classes, plus one additional feature per class (bias term). In total, the model estimates 1504 coefficients in the case of the Thetford mines data, while for the Houston and Zurich summer cases, it deals with 5775 and 3294 coefficients, respectively.

Results: The results are reported in Fig. 9, comparing the regularization paths for the four regularizers and the three data sets presented previously. The graphs can be read as a ROC curve: the most desirable situation would be a classifier with both accuracy and little active features, i.e., a score close to the top-left corner. The $\ell_{2}$ model shows no variation on the sparsity axis (all of the coefficients are active) and very little variability on the accuracy one: it is therefore represented by a single green dot. It is remarkably accurate but is the less compact model since it has all of the coefficients active. Employing the $\ell_{1}$ regularizer (red line), as it is mainly done in the literature of sparse classification, achieves a sharp decrease in the number of active coefficients but at the price of a steep decrease in performances of the classifier. When using 100 active coefficients, the $\ell_{1}$ model suffers from a $20 \%$ drop in performance, and a trend is observed in all of the experiments.

Using the nonconvex regularizers provides the best of both worlds: the $\ell_{p}$ regularizer (black line with “ $\square$ ” markers) in particular and also the LSP regularizer (blue line with " $\times$ " markers) achieve improvements of about $15 \%-20 \%$ with respect to the $\ell_{1}$ model. More stable results along the regularization path are 
observed: the nonconvex regularizers are less biased than the $\ell_{1}$ norm in classification and achieve competitive performances with respect to the (nonsparse) $\ell_{2}$ model with a fraction of the features (around 1\%-2\%). Note that the models of all experiments were initialized with the $\mathbf{0}$ vector. This is sensible for the nonconvex problem since all of the regularizations discussed in this paper (even $\ell_{2}$ ) tend to shrink the model toward this point. By initializing at $\mathbf{0}$ for nonconvex regularization, we simply promote a local solution not too far from this neutral point. The initialization can be seen as an additional regularization. Moreover, the experiments show that the nonconvexity leads to state-of-the-art performance.

\section{SPARSE LinEAR UNMIXING}

In this section, we express the sparse linear unmixing problem in the same optimization framework as (7). We discuss the advantage of using nonconvex optimization. The performances of the $\ell_{2}, \ell_{1}$, nonconvex $\ell_{p}$, and LSP regularization terms are then compared on a simulated example using real reflectance spectra (as in [18]).

\section{A. Model}

Sparse linear unmixing can be expressed as the following optimization problem:

$$
\min _{\boldsymbol{\alpha} \geq 0} \frac{1}{2}\|\mathbf{y}-\mathbf{D} \boldsymbol{\alpha}\|_{2}^{2}+\lambda R(\boldsymbol{\alpha})
$$

where $\mathbf{y}$ is a noisy spectrum observed and $\mathbf{D}$ is a matrix containing a dictionary of spectra (typically a spectral library). This formulation adds a positivity constraint to the vector $\boldsymbol{\alpha}$ w.r.t. problem (7). In practice, (8) can be reformulated as the following unconstrained optimization problem:

$$
\min _{\boldsymbol{\alpha}} \frac{1}{2}\|\mathbf{y}-\mathbf{D} \boldsymbol{\alpha}\|_{2}^{2}+\lambda R(\boldsymbol{\alpha})+\imath_{\boldsymbol{\alpha} \geq 0}
$$

where $\imath_{\boldsymbol{\alpha} \geq 0}$ is the indicator function that has a value of $+\infty$ when one of the components of $\boldsymbol{\alpha}$ is $<0$ and a value of 0 when it is in the positive orthant. By supposing that $\imath_{\boldsymbol{\alpha} \geq 0}$ is equivalent to $\lambda \imath_{\boldsymbol{\alpha}>0}, \forall \lambda>0$, we can gather the last two terms into $\tilde{R}(\boldsymbol{\alpha})=R(\boldsymbol{\alpha})+\imath_{\boldsymbol{\alpha} \geq 0}$, thus leading to a problem similar to (7). All of the optimization procedures discussed previously can therefore be used for this reformulation as long as the proximal operator w.r.t. $\tilde{R}(\cdot)$ can be computed efficiently. The proximal operator for all of the regularization terms in Table I with additional positivity constraints can be obtained by an orthogonal projection on the positive orthant followed by the proximal of $R$

$$
\operatorname{prox}_{\lambda R+\imath_{\alpha \geq 0}}(\mathbf{v})=\operatorname{prox}_{\lambda R}(\max (\mathbf{v}, 0))
$$

where $\max (\mathbf{v}, 0)$ is taken componentwise. This shows that we can use the same algorithm as in the classification experiments of Section III since we have an efficient proximal operator.

We know that in practice the true components of alpha are sparse (only a few component in each spectrum). To promote this sparsity we use a nondifferentiable regularization term $\mathrm{R}(\boldsymbol{\alpha})$ in equation (8). Therefore, in the following, we investigate the use of nonconvex regularization for linear unmixing. We focus on problem (8), but a large part of the unmixing literature works with an additional constraint of sum to 1 for the $\boldsymbol{\alpha}$ coefficients. This additional prior can sometimes reflect a physical measure and adds some information to the optimization problem. In our framework, this constraint can make the direct computation of the proximal operator nontrivial. In this case, it is more interesting to use multiple splitting instead of one and to use other algorithms such as generalized FBS [70] or ADMM, which has already been used for remote sensing applications [71].

\section{B. Numerical Experiments}

In the unmixing application, we consider an example simulated using the USGS spectral library ${ }^{6}$ : from the library, we extract 23 spectra corresponding to different materials (by keeping spectra with less than $15^{\circ}$ angular distance to each other). Using these 23 base spectra, we simulate mixed pixels by creating random linear combinations of $n_{\text {act }} \leq 23$ endmembers. The random weight of the active components is obtained using a uniform random generation in $[0,1]$ (leading to weights that do not sum to 1$)$. We then add to the resulting signatures some Gaussian noise $n \sim \mathcal{N}\left(0, \sigma^{2}\right)$. For each numerical experiment, we solve the unmixing problem by least squares with the four regularizers of Table I: $\ell_{2}, \ell_{1}, \ell_{p}$, and LSP. An additional approach that consists in performing a hard thresholding on the positive least square solution (so the $\ell_{2}$ ) has also been investigated (named "LS+threshold" hereafter). As for the previous example on classification, we calculate the unmixing performance on a regularization path, i.e., a series of values of the regularization parameter $\lambda$ in (8), with $\lambda=\left[10^{-5}, \ldots, 10^{3}\right]$. We assess the success of the unmixing by the model error $\left\|\boldsymbol{\alpha}-\boldsymbol{\alpha}_{\text {true }}\right\|^{2}$. We repeat the simulation 50 times to account for different combinations of the original elements of the dictionary: all results reported are averages over those 50 simulations.

First, we compare the different regularization schemes for different noise levels (Fig. 10). We set $n_{\text {act }}=3$ and report the model error along the regularization path (varying $\lambda$ ) at the top row of Fig. 10. At the bottom row, we report the model error as a function of the number of selected components, again along the same regularization path. We observe that the nonconvex strategies achieve the lowest errors (triangle-shaped markers) on low and medium noise levels but also that $\ell_{p}$ seems to be more robust to noise. The $\ell_{1}$ norm also achieves good results, particularly in high-noise situations. Regarding the error achieved per level of sparsity (represented at the bottom row of Fig. 10), we observe that the nonconvex regularizers achieve far better reconstruction errors, particularly around the right number of active coefficients (here $n_{\text {act }}=3$ ). On average, the best results are obtained by the LSP and $\ell_{p}$ regularization. Note that the $\ell_{1}$ regularizer needs a larger number of active components in order to achieve good model reconstruction (on the order of 9 when the actual number of coefficients is 3 ). The $\mathrm{LS}+$ threshold approach seems to work well for component selection but leads to an important decrease in accuracy of the model.

In order to evaluate the ability of a method to estimate a good model and select the good active components at the same time, we run simulations with a fixed noise level $\sigma=0.05$ but

${ }^{6}$ The data set can be downloaded from http://www.lx.it.pt/ bioucas/. 

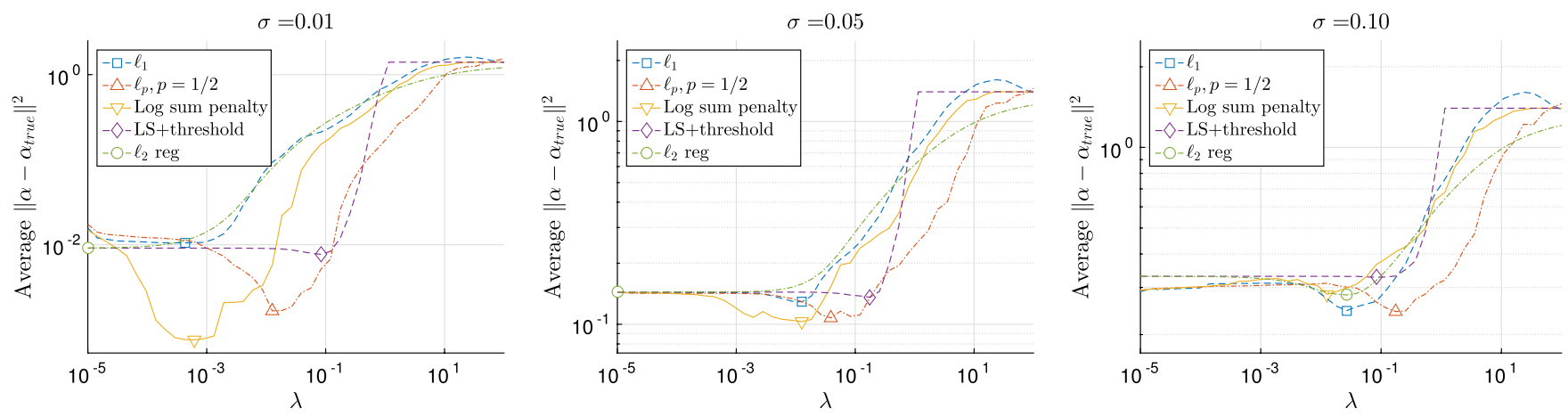

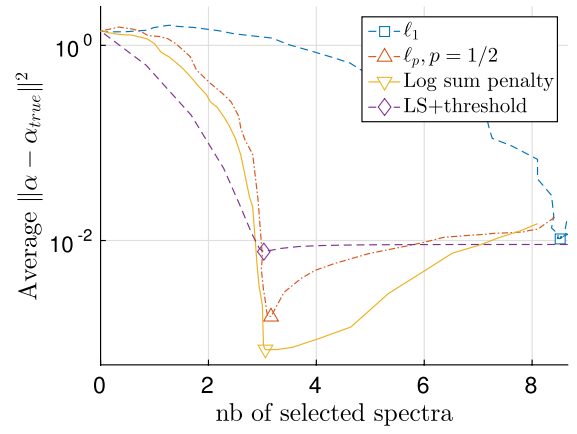

(a)

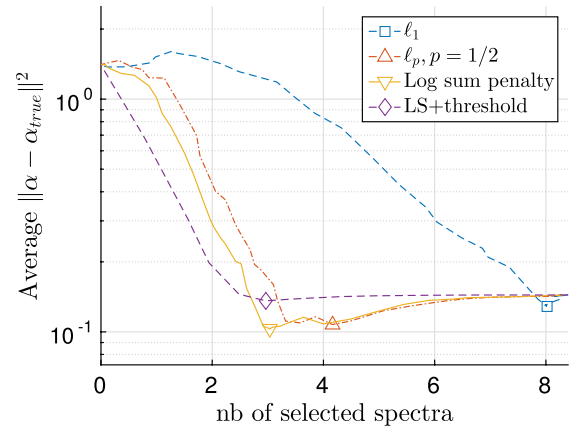

(b)

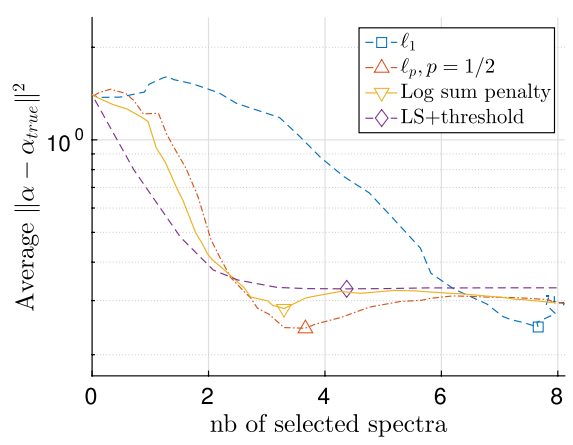

(c)

Fig. 10. Linear unmixing results on the simulated hyperspectral data set. Each column represents a different noise level. (a) $\sigma=0.01$. (b) $\sigma=0.05$. (c) $\sigma=0.10$. Model error $\left\|\boldsymbol{\alpha}-\boldsymbol{\alpha}_{\text {true }}\right\|^{2}$ is plotted as a function either of the regularization parameter $\lambda$ (top row) or of the number of active coefficients of the final solution (bottom row). The marker shows the best performances of each regularization strategy.

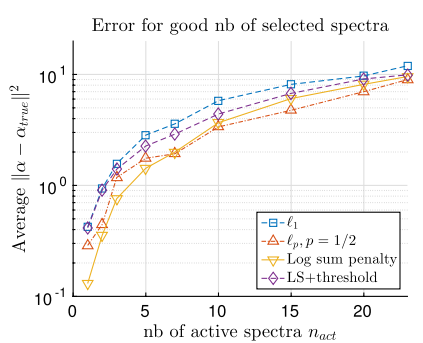

(a)

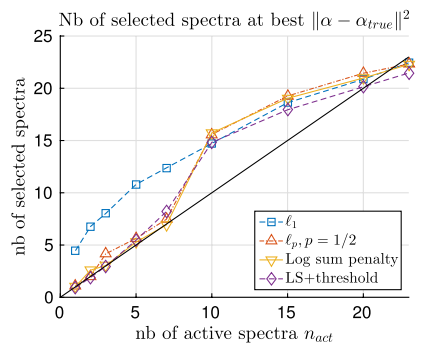

(b)

Fig. 11. Linear unmixing results on the simulated hyperspectral data set for increasing number of active spectra in the mixture: (a) model error for the best solution with the number of selected spectra closest to $n_{\text {act }}$ and (b) number of selected spectra for the model with the lowest error.

for a varying number of true active components $n_{\text {act }}$, from 1 to 23 . In this configuration, we first find for all regularizations the smallest $\lambda$ that leads to the correct number of selected component $n_{\text {sel }}=n_{\text {act }}$. The average model error as a function of $n_{\text {act }}$ is reported in Fig. 11(a). We can see that the nonconvex regularization leads to better performances when the correct number of spectra is selected (compared to $\ell_{1}$ and LS+threshold). In Fig. 11(b), we report the number of selected components as a function of the true number of active components when the model error is minimal. We observe that nonconvex regularization manages to both select the correct components and estimate a good model when a small number of components are active $\left(n_{\text {act }} \leq 10\right)$ but also that it fails (as $\ell_{1}$ does) for large numbers of active components. This result illustrates the fact that nonconvex regularization is more aggressive in terms of sparsity and obviously performs best when sparsity is truly needed.

\section{CONCLUSION}

In this paper, we have presented a general framework for nonconvex regularization in remote sensing image processing. We have discussed different ways to promote sparsity and avoid the bias when sparsity is required via the use of nonconvex regularizers. We have applied the proposed regularization schemes to problems of image classification and linear unmixing: in all scenarios, we have showed that nonconvex regularization leads to the best performances when accounting for both sparsity and quality of the final product. Nonconvex regularizers promote compact solutions but without the bias (and the decrease in performance) related to nondifferentiable convex norms such as the popular $\ell_{1}$ norm.

Nonconvex regularization is a flexible and general framework that can be applied to every regularized processing scheme: keeping this in mind, we have also provided a toolbox to the community to apply nonconvex regularization to a wider number of problems. The toolbox can be accessed in Github (see the Appendix for a description of the toolbox).

\section{APPENDIX \\ OPTIMIZATION TOOLBOX}

To promote the use of nonconvex regularization in the remote sensing community, we provide the reader with a simple to use MATLAB/Octave generic optimization toolbox. The code provides a generic solver (complete rewriting of GIST) for problem (7) that is able to handle a number of regularization terms (at least all of the terms in Table I) and any differentiable data fitting term $L$. We provide several functions for performing multiclass classification tasks such as SVM, logistic regression, 
and calibrated hinge loss. For linear unmixing, we provide the least squares loss, but extension to other more robust data fitting terms can be performed easily. For instance, performing unmixing with the Huber loss [72] would require the change of two lines in function "gist_least.m," i.e., the computation of the Huber loss and its gradient. The toolbox is available at https://github.com/rflamary/nonconvex-optimization. It is freely available as a community project, and we welcome contributions.

\section{ACKNOWLEDGMENT}

The authors would like to thank the IEEE GRSS Image Analysis and Data Fusion Technical Committee (IADFTC) for organizing and making available the data of the Data Fusion Contests 2013 and 2104. For the contest 2014, they also acknowledge Telops Inc. (Québec, Canada) for acquiring and providing the Thetford mines data, the Centre de Recherche Public Gabriel Lippmann and Dr. M. Schlerf for their contribution on the Hyper-Cam LWIR sensor, Dr. M. De Martino (University of Genoa, Genova, Italy) for providing the ground truth and Dr. M. Shimoni (Royal Military Academy, Belgium) for organizing the contest. For the 2013 contest, they also acknowledge the Hyperspectral Image Analysis Group and the NSF Funded Center for Airborne Laser Mapping (NCALM) at the University of Houston, Houston, TX, USA, for providing the Houston data. Finally, the authors acknowledge Dr. Volpi and Dr. Longbotham for making the Zurich summer data available, and Dr. Iordache and Dr. Bioucas-Dias for sharing the USGS library used in the unmixing experiment.

\section{REFERENCES}

[1] G. Camps-Valls, D. Tuia, L. Gómez-Chova, S. Jimenez, and J. Malo, Remote Sensing Image Processing, ser. Synthesis Lectures on Image, Video, and Multimedia Processing. Vermont, Vic., Australia: Morgan and Claypool, 2011.

[2] G. Camps-Valls, D. Tuia, L. Bruzzone, and J. Benediktsson, "Advances in hyperspectral image classification: Earth monitoring with statistical learning methods," IEEE Signal Process. Mag., vol. 31, no. 1, pp. 45-54, Jan. 2014.

[3] G. Camps-Valls and L. Bruzzone, "Kernel-based methods for hyperspectral image classification," IEEE Trans. Geosci. Remote Sens., vol. 43, no. 6 , pp. 1351-1362, Jun. 2005.

[4] G. Mountrakis, J. Ima, and C. Ogole, "Support vector machines in remote sensing: A review," ISPRS J. Photogramm. Remote Sens., vol. 66, no. 3, pp. 247-259, 2011

[5] J. Verrelst et al., "Machine learning regression algorithms for biophysical parameter retrieval: Opportunities for Sentinel-2 and -3," Remote Sens. Environ., vol. 118, pp. 127-139, 2012.

[6] M. Belkin, I. Matveeva, and P. Niyogi, "On manifold regularization," in Proc. 10th Int. Workshop AISTAT, Bonn, Germany, 2005, pp. 17-24.

[7] L. Gómez-Chova, G. Camps-Valls, J. Muñoz-Marí, and J. Calpe, "Semisupervised image classification with Laplacian support vector machines," IEEE Geosci. Remote Sens. Lett., vol. 5, no. 4, pp. 336-340, 2008.

[8] M. A. Bencherif, J. Bazi, A. Guessoum, N. Alajlan, F. Melgani, and H. Alhichri, "Fusion of extreme learning machine and graph-based optimization methods for active classification of remote sensing images," IEEE Geosci. Remote Sens. Lett., vol. 12, no. 3, pp. 527-531, Mar. 2015.

[9] W. Zhangyang, N. Nasrabadi, and T. S. Huang, "Semisupervised hyperspectral classification using task-driven dictionary learning with Laplacian regularization," IEEE Trans. Geosci. Remote Sens., vol. 53, no. 3, pp. 1161-1173, Mar. 2015.

[10] X. Sun, N. Nasrabadi, and T. Tran, "Task-driven dictionary learning for hyperspectral image classification with structured sparsity constraints," IEEE Trans. Geosci. Remote Sens., vol. 53, no. 8, pp. 4457-4471, Aug. 2015.
[11] S. T. Tu, J. Y. Chen, W. Yang, and H. Sun, "Laplacian eigenmapsbased polarimetric dimensionality reduction for SAR image classification," IEEE Trans. Geosci. Remote Sens., vol. 50, no. 1, pp. 170-179, Jan. 2012

[12] D. Tuia, M. Volpi, M. Trolliet, and G. Camps-Valls, "Semisupervised manifold alignment of multimodal remote sensing images," IEEE Trans. Geosci. Remote Sens., vol. 52, no. 12, pp. 7708-7720, Dec. 2014.

[13] D. L. Donoho, "Compressed sensing," IEEE Trans. Inf. Theory, vol. 52, no. 4, pp. 1289-1306, Apr. 2006.

[14] M. Fauvel, Y. Tarabalka, J. A. Benediktsson, J. Chanussot, and J. C. Tilton, "Advances in spectral-spatial classification of hyperspectral images," Proc. IEEE, vol. 101, no. 3, pp. 652-675, Mar. 2013.

[15] D. Tuia, M. Volpi, M. Dalla Mura, A. Rakotomamonjy, and R. Flamary, "Automatic feature learning for spatio-spectral image classification with sparse SVM," IEEE Trans. Geosci. Remote Sens., vol. 52, no. 10, pp. 6062-6074, Oct. 2014.

[16] C. Romero, A. und Gatta, and G. Camps-Valls, "Unsupervised deep feature extraction for remote sensing image classification," IEEE Trans. Geosci. Remote Sens., vol. 54, no. 3, pp. 1349-1362, 2016.

[17] M. Campos-Taberner et al., "Processing of extremely high resolution LiDAR and optical data: Outcome of the 2015 IEEE GRSS Data Fusion Contest. Part A: 2D contest," IEEE J. Sel. Topics Appl. Earth Observ. Remote Sens., to be published.

[18] M.-D. Iordache, J. M. Bioucas-Dias, and A. Plaza, "Sparse unmixing of hyperspectral data," IEEE Trans. Geosci. Remote Sens., vol. 49, no. 6, pp. 2014-2039, Jun. 2011

[19] P. Tokarczyk, J. Wegner, S. Walk, and K. Schindler, "Features, color spaces, and boosting: New insights on semantic classification of remote sensing images," IEEE Trans. Geosci. Remote Sens., vol. 53, no. 1, pp. 280-295, Jan. 2014.

[20] M. Volpi and D. Tuia, "Dense semantic labeling with convolutional neural networks," IEEE Trans. Geosci. Remote Sens., to be published.

[21] J. Bioucas-Dias et al., "Hyperspectral unmixing overview: Geometrical, statistical, and sparse regression-based approaches," IEEE J. Sel. Topics Appl. Earth Observ., vol. 5, no. 2, pp. 354-379, Apr. 2012.

[22] Q. Qu, N. Nasrabadi, and T. Tran, "Abundance estimation for bilinear mixture models via joint sparse and low-rank representation," IEEE Trans. Geosci. Remote Sens., vol. 52, no. 7, pp. 4404-4423, Jul. 2014.

[23] M.-D. Iordache, J. Bioucas-Dias, and A. Plaza, "Collaborative sparse regression for hyperspectral unmixing," IEEE Trans. Geosci. Remote Sens., vol. 52, no. 1, pp. 341-354, Jan. 2014.

[24] Y. Chen, N. Nasrabadi, and T. Tran, "Hyperspectral image classification using dictionary-based sparse representation," IEEE Trans. Geosci. Remote Sens., vol. 49, no. 10, pp. 3973-3985, Oct. 2011.

[25] K. Tan, S. Zhou, and Q. Du, "Semisupervised discriminant analysis for hyperspectral imagery with block-sparse graph," IEEE Geosci. Remote Sens. Lett., vol. 12, no. 8, pp. 1765-1769, Aug. 2015.

[26] B. Song et al., "Remotely sensed image classification using sparse representations of morphological attribute profiles," IEEE Trans. Geosci. Remote Sens., vol. 52, no. 8, pp. 5122-5136, Aug. 2014.

[27] D. Tuia, N. Courty, and R. Flamary, "Multiclass feature learning for hyperspectral image classification: Sparse and hierarchical solutions," ISPRS J. Int. Soc. Photo. Remote Sens., vol. 105, pp. 272-285, 2015.

[28] X. X. Zhu and R. Bamler, "Super-resolution power and robustness of compressive sensing for spectral estimation with application to spaceborne tomographic SAR," IEEE Trans. Geosci. Remote Sens., vol. 50, no. 1, pp. 247-258, Jan. 2012.

[29] H. Hongxing, J. Bioucas-Dias, and V. Katkovnik, "Interferometric phase image estimation via sparse coding in the complex domain," IEEE Trans. Geosci. Remote Sens., vol. 53, no. 5, pp. 2587-2602, May 2015.

[30] S. Li and B. Yang, "A new pan-sharpening method using a compressed sensing technique," IEEE Trans. Geosci. Remote Sens., vol. 49, no. 2, pp. 738-746, Feb. 2011.

[31] D. L. Donoho and P. B. Stark, "Uncertainty principles and signal recovery," SIAM J. Appl. Math., vol. 49, no. 3, pp. 906-931, 1989.

[32] D. L. Donoho and M. Elad, "Optimally sparse representation in general (nonorthogonal) dictionaries via 1 minimization," Proc. Nat. Acad. Sci., vol. 100, no. 5, pp. 2197-2202, 2003.

[33] E. J. Candès, "The restricted isometry property and its implications for compressed sensing," Comptes Rendus Math., vol. 346, no. 9, pp. 589-592, 2008.

[34] P. L. Combettes and V. R. Wajs, "Signal recovery by proximal forward-backward splitting," Multiscale Model. Simul., vol. 4, no. 4, pp. 1168-1200, 2005.

[35] S. Mosci, L. Rosasco, M. Santoro, A. Verri, and S. Villa, "Solving structured sparsity regularization with proximal methods," in Machine Learning and Knowledge Discovery in Databases. New York, NY, USA: Springer-Verlag, 2010, pp. 418-433. 
36] P. L. Combettes and J.-C. Pesquet, "Proximal thresholding algorithm for minimization over orthonormal bases," SIAM J. Optim., vol. 18, no. 4 pp. 1351-1376, 2007.

37] R. Tibshirani, "Regression shrinkage and selection via the Lasso," J. Roy. Statist. Soc. B, Methodol., vol. 58, pp. 267-288, 1994.

[38] T. Hastie, S. Rosset, R. Tibshirani, and J. Zhu, "The entire regularization path for the support vector machine," J. Mach. Learn. Res., vol. 5, pp. 1391-1415, 2004

[39] D. L. Donoho and B. F. Logan, "Signal recovery and the large sieve," SIAM J. Appl. Math., vol. 52, no. 2, pp. 577-591, 1992

[40] E. J. Candes, M. B. Wakin, and S. P. Boyd, "Enhancing sparsity by reweighted $\ell_{1}$ minimization," J. Fourier Anal. Appl., vol. 14, no. 5/6, pp. 877-905, 2008.

[41] T. Zhang, "Analysis of multi-stage convex relaxation for sparse regularization," J. Mach. Learn. Res., vol. 11, pp. 1081-1107, 2010.

[42] J. Sigurdsson, M. Ulfarsson, and J. Sveinsson, "Hyperspectral unmixing with $\ell_{q}$ regularization," IEEE Trans. Geosci. Remote Sens., vol. 52, no. 11, pp. 6793-6806, Nov. 2014.

[43] Y. Qian, S. Jia, J. Zhou, and A. Robles-Kelly, "Hyperspectral unmixing via $\ell_{1 / 2}$ sparsity-constrained nonnegative matrix factorization," IEEE Trans. Geosci. Remote Sens., vol. 49, no. 11, pp. 4282-4297, Nov. 2011.

[44] W. Wang and Y. Qian, "Adaptive $\ell_{1 / 2}$ sparsity-constrained NMF with half-thresholding algorithm for hyperspectral unmixing," IEEE J. Sel. Topics Appl. Earth Observ., vol. 8, no. 6, pp. 2618-2631, Jun. 2015

[45] M.-H. Wei, J. McClellan, and W. Scott, "Estimation of the discrete spectrum of relaxations for electromagnetic induction responses using $\ell_{p}$-regularized least squares for $0<p<1$," IEEE Geosci. Remote Sens. Lett., vol. 8, no. 2, pp. 233-237, Mar. 2011.

[46] J. Zhang, P. Zhong, Y. Chen, and S. Li, " $\ell_{1 / 2}$-regularized deconvolution network for the representation and restoration of optical remote sensing images," IEEE Trans. Geosci. Remote Sens., vol. 52, no. 5, pp. 2617-2627, May 2014

[47] S. Jia, X. Zhang, and Q. Li, "Spectral-spatial hyperspectral image classification using $\ell_{1 / 2}$ regularized low-rank representation and sparse representation-based graph cuts," IEEE J. Sel. Topics Appl. Earth Observ., vol. 8, no. 6, pp. 2473-2484, Jun. 2015.

[48] P. Gong, C. Zhang, Z. Lu, J. Z. Huang, and J. Ye, "A general iterative shrinkage and thresholding algorithm for non-convex regularized optimization problems," in Proc. ICML, Atlanta, GA, USA, 2013.

[49] O. Bousquet and A. Elisseeff, "Stability and generalization," J. Mach. Learn. Res., vol. 2, pp. 499-526, 2002.

50] B. Schölkopf, C. J. Burges, and A. J. Smola, Advances in Kernel Methods: Support Vector Learning. Cambridge, MA, USA: MIT Press, 1998.

[51] S. Bourguignon, J. Ninin, H. Carfantan, and M. Mongeau, "Exact sparse approximation problems via mixed-integer programming: Formulations and computational performance," IEEE Trans. Signal Proc., vol. 64, no. 6, pp. 1405-1419, Mar. 2016.

52] Y. C. Pati, R. Rezaiifar, and P. Krishnaprasad, "Orthogonal matching pursuit: Recursive function approximation with applications to wavelet decomposition," in Conf. Rec. IEEE 27th Asilomar Conf. Signals, Syst. Comput., 1993, pp. 40-44.

[53] S. Chen, C. F. Cowan, and P. M. Grant, "Orthogonal least squares learning algorithm for radial basis function networks," IEEE Trans. Neural Netw., vol. 2, no. 2, pp. 302-309, Mar. 1991.

[54] B. Efron et al., "Least angle regression," Ann. Statist., vol. 32, no. 2, pp. 407-499, 2004.

[55] P. L. Combettes and J.-C. Pesquet, "Proximal splitting methods in signal processing," in Fixed-Point Algorithms for Inverse Problems in Science and Engineering. New York, NY, USA: Springer-Verlag, 2011, pp. 185-212.

[56] H. Zou, "The adaptive Lasso and its oracle properties," J. Amer. Statist. Assoc., vol. 101, no. 476, pp. 1418-1429, 2006.

57] J. Fan and R. Li, "Variable selection via nonconcave penalized likelihood and its oracle properties," J. Amer. Statist. Assoc., vol. 96, no. 456, pp. 1348-1360, 2001.

[58] C. Zhang, "Nearly unbiased variable selection under minimax concave penalty," Ann. Statist., vol. 38, no. 2, pp. 894-942, 2010.

[59] H. Attouch, J. Bolte, P. Redont, and A. Soubeyran, "Proximal alternating minimization and projection methods for nonconvex problems: An approach based on the Kurdyka-Lojasiewicz inequality," Math. Oper. Res. vol. 35, no. 2, pp. 438-457, 2010.

60] Z. Xu, X. Chang, F. Xu, and $\mathrm{H}$. Zhang, " $L_{1 / 2}$ regularization: A thresholding representation theory and a fast solver," IEEE Trans. Neural Netw. Learn. Syst., vol. 23, no. 7, pp. 1013-1027, Jul. 2012.

61] E. Chouzenoux, J.-C. Pesquet, and A. Repetti, "A block coordinate variable metric forward-backward algorithm," in J. Global Optimiz., New York, NY, USA: Springer-Verlag, 2013, pp. 1-29.
62] A. Rakotomamonjy, R. Flamary, and G. Gasso, "DC proximal Newton for non-convex optimization problems," IEEE Trans. Neural Netw. Learn. Syst., vol. 27, no. 3, pp. 636-647, Mar. 2016.

63] L. Laporte, R. Flamary, S. Canu, S. Déjean, and J. Mothe, "Nonconvex regularizations for feature selection in ranking with sparse SVM," IEEE Trans. Neural Netw. Learn. Syst., vol. 25, no. 6, pp. 1118-1130, Jun. 2014.

[64] G. Gasso, A. Rakotomamonjy, and S. Canu, "Recovering sparse signals with a certain family of nonconvex penalties and dc programming," IEEE Trans. Signal Process., vol. 57, no. 12, pp. 4686-4698, Dec. 2009.

[65] J. Friedman, T. Hastie, and R. Tibshirani, "Regularization path for generalized linear models via coordinate descent," J. Statist. Softw., vol. 33, pp. 1-122, 2010.

[66] W. Liao et al., "Processing of thermal hyperspectral and digital color cameras: Outcome of the 2014 Data Fusion Contest," IEEE J. Sel. Topics Appl. Earth Observ. Remote Sens., vol. 8, no. 6, pp. 2984-2996, Jun. 2015.

[67] F. Pacifici, Q. Du, and S. Prasad, "Report on the 2013 IEEE GRSS Data Fusion Contest: Fusion of hyperspectral and LiDAR data," IEEE Remote Sens. Mag., vol. 1, no. 3, pp. 36-38, Jun. 2013.

[68] M. Volpi and V. Ferrari, "Semantic segmentation of urban scenes by learning local class interactions," in Proc. IEEE/CVF CVPRW Earthvis., 2015, pp. 1-9.

[69] P. Felzenszwalb and D. Huttenlocher, "Efficient graph-based image segmentation," Int. J. Comput. Vis., vol. 59, no. 2, pp. 167-181, Sep. 2004.

[70] H. Raguet, J. Fadili, and G. Peyré, "A generalized forward-backward splitting," SIAM J. Imaging Sci., vol. 6, no. 3, pp. 1199-1226, 2013.

[71] M.-D. Iordache, J. M. Bioucas-Dias, and A. Plaza, "Total variation spatial regularization for sparse hyperspectral unmixing," IEEE Trans. Geosci. Remote Sens., vol. 50, no. 11, pp. 4484-4502, Nov. 2012.

[72] P. J. Huber et al., "Robust estimation of a location parameter," Ann. Math. Statist., vol. 35, no. 1, pp. 73-101, 1964

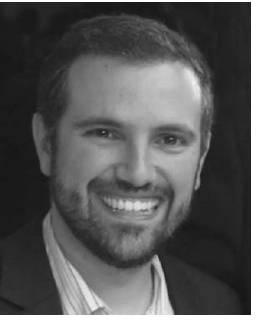

Devis Tuia (S'07-M'09-SM'15) received the Ph.D degree in environmental sciences from the University of Lausanne, Lausanne, Switzerland, in 2009.

He was then a Postdoctoral Researcher with the University of València, València, Spain, the University of Colorado, Boulder, CO, USA, and EPFL Lausanne, Lausanne. Since 2014, he has been an Assistant Professor with the University of Zurich, Zurich, Switzerland. His research focuses on information extraction and data fusion of remote sensing images using machine learning algorithms.

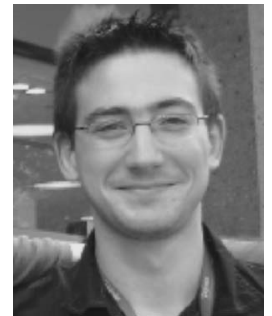

Rémi Flamary received the Dipl.-Ing. degree in electrical engineering and the M.S. degree in image processing from the Institut National de Sciences Appliquées de Lyon, Lyon, France, in 2008 and the Ph.D. degree from the University of Rouen, Rouen, France, in 2011.

He is an Assistant Professor with Université Côte d'Azur (UCA), Nice, France, and he has been a member of Lagrange Laboratory/Observatoire de la Côte d'Azur, Nice, since 2012. His current research ing, and image processing. interests involve signal processing, machine learn-

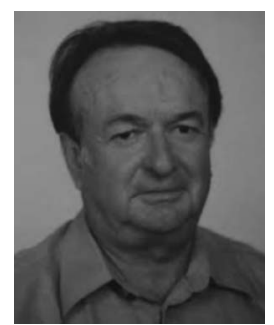

Michel Barlaud (M'85-SM'95) received the "Agregation de Physique" and "These d'état" from the University of Paris XI, Orsay, France, in 1976 and 1983, respectively.

He has been a Full Professor with Université Côte d'Azur, Nice, France, since 1984 and a Senior Member of the Institut Universitaire de France, Paris, France. He joined the I3S laboratory in 1989. His current research interest involves machine learning and convex optimization for image and genomic applications

Prof. Barlaud has cofounded and served as an Associate Editor of the IEEE TRANSACTIONS ON IMAGE PROCESSING. He has served on more than $200 \mathrm{Ph}$.D. committees worldwide, and he was a member of the Technical Committee of the IEEE Signal Processing Society. 